\title{
Radiology department preventive and control measures and work plan during COVID-19 epidemic-experience from Wuhan
}

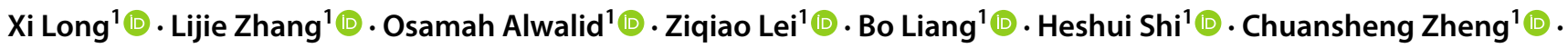 \\ Fan Yang ${ }^{1}$ (1)
}

Received: 23 July 2020 / Revised: 22 January 2021 / Accepted: 3 February 2021 / Published online: 19 February 2021

(c) The Author(s), under exclusive licence to Springer Nature Singapore Pte Ltd. part of Springer Nature 2021

\begin{abstract}
Since the outbreak of the coronavirus disease 2019 (COVID-19), it had rapidly spread to the whole world and seriously threatened the global health. Imaging examination plays an important role in the clinical diagnosis of this disease, which leads to the high infection risk of the medical staff in the radiology department. In this review, the authors thoroughly summed up the experience in the management and operation of radiology department and shared their experience of the protective and control strategies and work plan during the epidemic, including but not limited to the management framework of the radiology department, the environment and layout in the department, the requirements for protection of different posts and the equipment, as well as the essential diagnosis of COVID-19. It is worth noting that the main goal of the radiology department in every country is to complete the radiology examination safely and make an accurate diagnosis of COVID-19 patients.
\end{abstract}

Keywords Coronavirus · COVID-2019 · Diagnosis $\cdot$ SARS-CoV-2 $\cdot$ Radiology $\cdot$ Occupational health

\section{Abbreviations \\ COVID-19 Coronavirus disease 2019 \\ CSG The Coronavirus Study Group \\ RT-PCR Reverse-transcription polymerase chain reaction}

SARS-CoV-2 Severe acute respiratory syndrome coronavirus 2

\section{Introduction}

Since the outbreak of the coronavirus disease 2019 (COVID19) in December 2019, it had rapidly spread to the whole world and seriously threatened the global health [1]. By April 7, 2020, a total of 83,096 patients had been diagnosed

Xi Long and Lijie Zhang contributed equally to this work.

Chuansheng Zheng

whxhchuansheng@126.com

Fan Yang

fyang@vip.163.com

1 Department of Radiology, Hubei Key Laboratory of Molecular Imaging, Union Hospital, Tongji Medical College, Huazhong University of Science and Technology, 1277 Jiefang Avenue, Wuhan 430022, China in China and 1,269,950 patients abroad. The Coronavirus Study Group (CSG) of the International Committee on Taxonomy of Viruses, which is responsible for developing the official classification of viruses and taxa naming (taxonomy) of the Coronaviridae family named the virus that caused the outbreak as severe acute respiratory syndrome coronavirus 2 (SARS-CoV-2), which is an RNA virus belonging to the $\beta$ genus of coronavirus. At present, the current data from the previous studies showed that the virus can be traced back to the Chinese Horseshoe Bat, which can be transmitted from human to human, infect medical staff, and spread rapidly in a certain range of communities [2,3]. Imaging examination plays an important role in the clinical diagnosis of this disease, and is also the most likely site for hospital cross infection. Nowadays, with the global pandemic expansion day by day, it is very important to effectively protect medical staff from infection, reduce hospital cross infection, and prepare for the large number of patients visiting radiology department for imaging examinations. With the gradual improvement of the domestic epidemic situation, we have thoroughly summed up our experience in the management and operation of radiology department during the epidemic situation, which is summarized in this article aiming to provide some helpful opinions and suggestions for the global pandemic prevention and control. 


\section{Work plan for the epidemic}

In this outbreak, the radiology department of Wuhan Union Hospital had to undertake clinical work in five battlefields: Union Hospital main campus (206 beds), the West Campus (810 beds), Jianghan temporary hospital (1523 beds), Cancer Center (850 beds), and Jianghan Development Zone temporary hospital (682 beds). During the peak period, the average daily examinations were 600-800. More than 30,000 cases with CT and/or DR examinations were diagnosed, and 2500 cases of difficult cases of COVID-19 were consulted.

Anti-COVID-19 leading group (Fig. 1) headed by director of the department was set up quickly in the early stage of the outbreak, with the following subdivisions: protection group and logistics support group, clinical diagnosis group, medical technology group, scientific research group, publicity group and teaching group. At the same time, the specific strategies in the department were developed based on national, provincial and municipal documents, as well as hospital epidemic prevention and control documents. All staff members were trained on infection prevention and control measures, so as to master the methods and skills of infection control and prevention; thereby everyone is aware, participating and strictly executing.

\section{Protective and control strategy during the epidemic}

\section{The environment and layout in the department}

1. According to the specific layout of radiology department in each hospital, polluted area (registration room, contrast enhancement preparation room, examination room, waiting area, delivery channel, elevator and patient toilet), semi-polluted area (channel between operation room and examination room), run-off zone (area between semi-polluted area and dressing room) and clean area (dressing room, office, diagnosis room, conference room, tea room, lounge and warehouse, etc.) were set up (Fig. 2). Accordingly, infection prevention and environment disinfection were carried out.

2. The dressing room was used for the staff to wear the protective equipment. After work, all kinds of protective equipment were to be removed in the semi-polluted

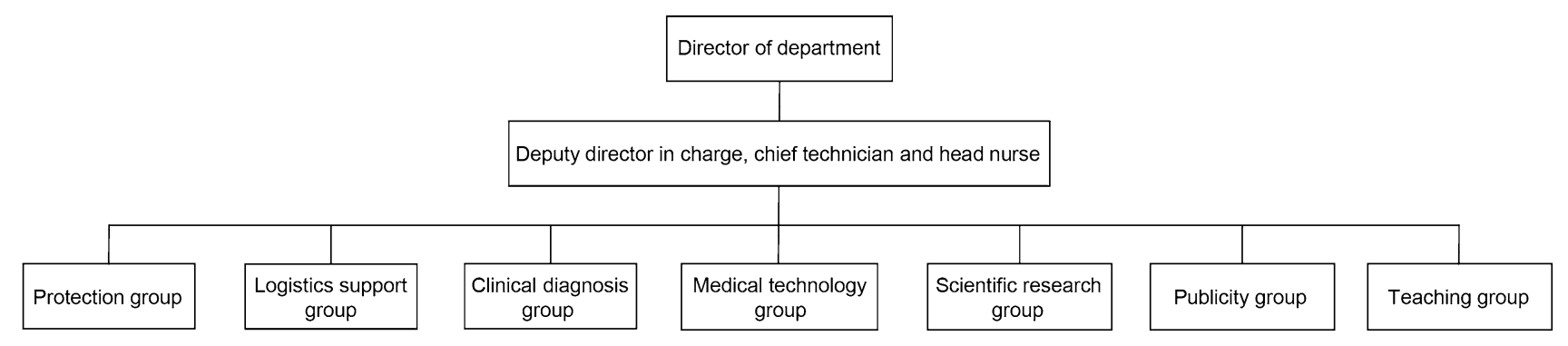

Fig. 1 The management framework of radiology department during the epidemic. Protection group: (1) by strictly following the "Technical Specifications for Hospital Isolation", they need to divide the space of the department into "three areas and two channels", which are polluted area, semi-polluted area, clean area, staff channel and patient channel according to the actual situation of the radiology department. (2) Develop dedicated inspection routes, dedicated channels, dedicated machines and dedicated booking desks. (3) Set up the prevention and control standards of COVID-19 in the department according to the standards of prevention and control in China. (4) Impart the knowledge of prevention, medical isolation technical standard, and the safe use of hand hygiene, wearing respirators, isolation clothing, and protective clothing. Otherwise, other training programs are also carried out to strengthen training for all staff in the front-line and key working areas. Logistics support group: Formulate the management and use methods and plans of medical materials in the department, and be responsible for the collection, distribution and registration of medical materials. More importantly, they are bound to ensure that the staff have basic living supplies. Clinical diagnosis group: (1) master the imaging features and protection knowledge of COVID-19, and provide timely reporting in the required area. (2) In case of suspected patients, they shall be treated according to the critical value process, and the senior radiologist shall be informed for review at the first time, the clinician shall be informed by phone, and the machine room shall be strictly disinfected at the same time. (3) Do a good job in personal protection and participate in the rescue of critical patients. Medical technology group: (1) examine patients according to the clinical requirements, learn knowledge of prevention, take preventive measures according to the level of post prevention and control, wear protective clothing and disinfect in the required areas. (2) Learn the imaging features of COVID-19, communicate with the diagnostic doctors in time, and report any problems timely. Scientific research group: (1) collect the clinical data thoroughly and share scientific research results with the world. (2) Summarize the epidemic prevention and control measures to provide experience for other regions. Publicity group: (1) do a good job in the popularization of COVID-19 health education and guide the general population to carry out self-protection. (2) Record the contacted people or things during the epidemic to facilitate infection control. Teaching group: (1) convey the management plan of the university and the hospital for students, while students are not allowed to work on the front line. (2) Help students who are affected by the epidemic and the delay of their study and practice by distant online teaching. COVID-19 coronavirus disease 2019 


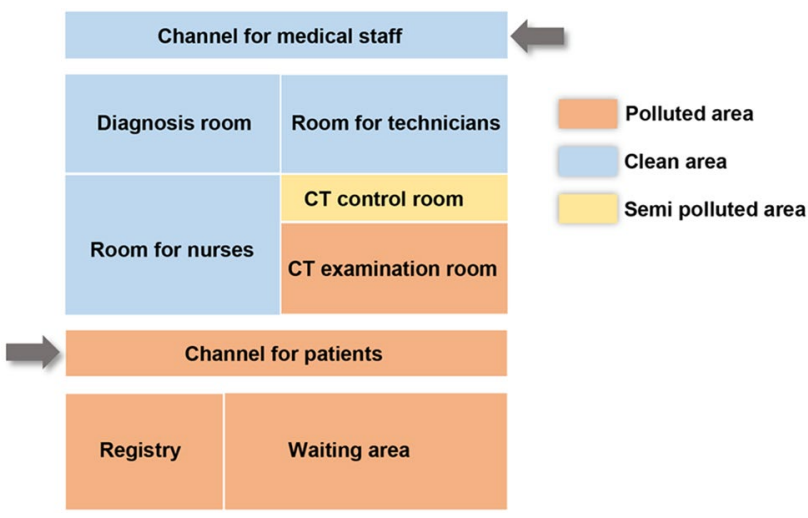

Fig. 2 The environment and layout in the department (two channels and three areas)

area, and work clothes are removed in the run-off zone. Personal cleaning, especially the hand hygiene is performed. Personal clothes are put on in the changing room.

3. The staff passage is separated from the patient passage. The unnecessary passages can be temporarily closed. The passage route of staff and patients should be divided according to the different areas. The staff protective equipment (including protective goggles or face shield (anti fog type), protective clothing, disposable shoe covers and double-layer disposable latex gloves) are limited for activities in the polluted and semi-polluted areas.

4. Quick-drying hand disinfectant is placed inside and outside the doors of all different areas, and the hands of personnel entering and leaving the doors are disinfected.
5. Special CT examination room, special machine for fever patients, suspected patients and confirmed patients were set up (Fig. 3) [4].

\section{Requirements for protection of different posts}

For protecting the registration personnel, the following instructions should be followed: (1) the registration personnel shall do a good job of personal protection according to the secondary protection: wear disposable protective cap, protective goggles or face shield (anti fog type), medical protective mask, protective clothing or isolation gown, disposable latex gloves, disposable shoe covers, and strictly implement hand hygiene (Table 1) [4, 5]. (2) Ask whether the patient has a history of fever or any other discomforts, and whether there was a travel history to an epidemic area or a contact history with suspected or confirmed COVID19 patients in the past two weeks. (3) Before submitting the application form, the patient should wear a mask, the registration staff should reduce the time talking with the patient, keep a proper distance from the patient, and inform the patient and his companions that they must wear masks before conducting the examination. (4) Ultraviolet rays and other measures can be used to disinfect the patient's examination order form and other papers. (5) Set up a separate report acquisition area for febrile patients, and set clear signs and guidelines to avoid multiple inquiries and movements of patients.

For radiology technicians, we suggest that: (1) personnel protection should be done according to the secondary protection: wear disposable protective cap, protective

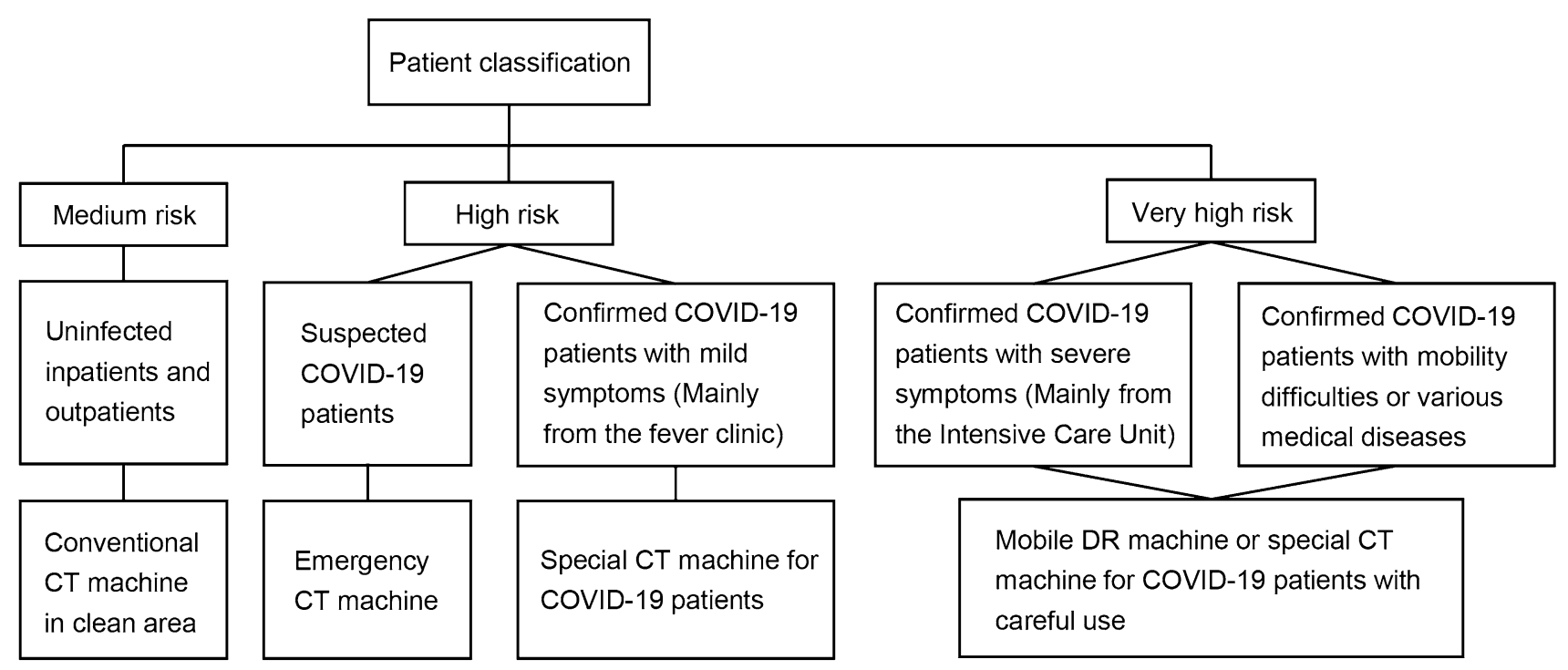

Fig. 3 Triage of patients with different risk levels 
goggles or shield (anti fog type), medical protective mask, protective clothing or isolation gown, disposable latex gloves, disposable shoe covers, and strictly implement hand hygiene [4, 5]. (2) Each area of CT unit, including control room (operation room), machine room and waiting area, needs to be equipped with air disinfection equipment, which should be disinfected regularly (twice or more times a day), and the room needs to be disinfected timely after the examination of high-risk patients. (3) Patients are required to wear masks for examination. For patients who can move the examination bed up and down by themselves, the technicians can control the check bed by the operating platform, to avoid close contact with patients, but must assess the patient's condition, to avoid accidental injury; for bed-ridden patients, it is necessary to cover the patients with blanket, and then move for the examination. (4) After each close contact with patients, wipe the hands with quick drying hand disinfectant immediately. If the door of machine room is not automatic, pay attention to the disinfection of the door handle. (5) It is better for the technician to work in pairs, separate the operator and the one who helps positioning the patients, try to ensure that the environment of the control room (operation room) is of low pollution, and that clinician accompanying the patient for examination should not enter the control room (operation room). (6) All protective equipment must be replaced when the technicians finish the work. Pay attention not to pollute the run-off zone and clean zone, and place the discarded protective equipment according to the specified site.

The requirements for radiologists are as follows: (1) do a good job of personal protection according to the first level of protection: wear surgical mask, disposable work cap and work cloth, strictly implement hand hygiene, and wear isolation clothes and disposable latex gloves if necessary [4, 5]. (2) Radiologists communicate with technicians by telephone or other means of communication, avoiding entering semipolluted areas. (3) Radiologists use telephone or other communication methods to communicate with patients and avoid direct face-to-face communication. (4) For the radiologist undertaking emergency and rescue task, personal protection should be done according to the secondary protection when contacting with patients in emergent situations.

The protocol of protection for the nurses is mainly divided into the following two parts: (1) for contrast-enhanced examinations or other circumstances that require the nurse to participate, personal protection shall be done well by the nurse according to the secondary protection: wearing disposable working cap, medical protective mask, protective goggles or face shield (anti fog type), work clothes, protective clothing, disposable shoe covers and double-layer disposable latex gloves, and strictly implement hand hygiene [4, 5]. (2) When the nurse inserts the indwelling canula or connects the 
high-pressure injector to the patient, they should avoid contacting with the patient's blood or body fluid, and conduct hand hygiene in time.

During the epidemic, the previous personnel management arrangement should be discontinued, the diagnostic radiologists should be arranged uniformly regardless of the subspecialty divisions, and the COVID-19 image reporting should be timely completed in each area. The number of technicians per shift should be reasonably arranged according to the patient flow regardless of subdivisions, and implement the system of changing technicians every day to ensure no fatigue and to reduce psychological pressure. Regular rotation and isolation system shall be implemented for all medical workers. Reasonably and evenly arrange the shifts and combine work with rest to fight the epidemic with high spiritual attitude.

\section{Protective measures for the equipment}

In strict accordance with the standards [6-9], clean and disinfect medical equipment, contaminated articles, surfaces and floors, as well as air.

1. CT equipment: after the patient's examination, the surface of the scanner should be immediately wiped and disinfected with 500-2000 mg/L chlorine-containing disinfectant. If it is not resistant to corrosion, it should be wiped and disinfected with $2 \%$ double-chain quaternary ammonium salt or $75 \%$ ethanol twice or more daily. Disposable disinfectant wipes can be used for cleaning and disinfection of one complete step. Disinfect at any time in case of contamination. When having visible pollutants, the disposable absorbent material shall be used first to remove the pollutants, followed by routine disinfection.

2. Other equipment: after the patient's examination, 250 $500 \mathrm{mg} / \mathrm{L}$ chlorine-containing disinfectant can be used to wipe and disinfect, or alcohol-containing disposable disinfectant wipes can be used to complete the cleaning and disinfection twice or more a day. Disinfect at any time in case of contamination. When there are visible pollutants such as vomitus, the disposable absorbent material should be used first to completely remove the pollutants and then disinfect. If no obvious pollutant, it can be wiped and disinfected with $500-2000 \mathrm{mg} / \mathrm{L}$ chlorine-containing disinfectant twice or more a day, and disinfect at any time in case of pollution.

For the examination rooms of suspected or confirmed patients, the circulating air sterilizer is used for continuous disinfection after the examination, and hydrogen peroxide air disinfector spray is used thereafter. Meanwhile, environmental ventilation should be strengthened.
Regarding the medical waste management, we suggest the following: (1) the protective equipment used by medical staff and all the waste of patients should be treated as infectious clinical waste, and the corresponding management regulations should be strictly followed, requiring doublelayer sealing, clear identification and airtight transport. (2) The infectious waste (including the articles contaminated by the patient's blood and body fluid, the domestic waste produced by the patients in the isolation, the disposable medical devices and supplies such as syringes, needles and other sharp tools must be put into the sharp tool box after use) should be put into the yellow medical waste collection bag, $3 / 4$ full, the bag shall be sprayed with $5000 \mathrm{mg} / \mathrm{L}$ chlorinecontaining disinfectant, the inner gooseneck-type seal, and the inner bag surface shall be sprayed with $5000 \mathrm{mg} / \mathrm{L}$ chlorine-containing disinfectant; the outer gooseneck-type seal is labeled with special identification, and the outer bag surface is sprayed with $5000 \mathrm{mg} / \mathrm{L}$ chlorine-containing disinfectant, and is placed in the temporary storage of medical waste in the department. (3) The cleaner or specialized medical waste collector shall wear personal protection (secondary protection) to collect infectious medical waste. Finish handover registration, airtight transfer and temporary storage of medical wastes in the hospital.

\section{The management of CT scanning technology and quality control}

(1) Before the examination, set up a CT machine to examine the suspected or confirmed cases, preferentially select mobile CT (if possible) or the one that can lift and lower the examination bed through the console, and have an independent control room (operation room). Air disinfection must be carried out for other rooms connected with the control room at the same time during the post examination disinfection if no independent control room. If the machine room adopts a central air conditioning system with fresh air, open the air supply and turn the volume of air conditioning to the maximum. If the machine room adopts the common central air conditioning, close the central air conditioning of the machine room and the operation room, and open the standby independent air conditioning. If there is no standby independent air conditioning, then open the central air conditioning after the examination and disinfection. To reduce the spread of the virus, a disposable bed sheet should be placed to separate the equipment from the patient. Arrange two technicians, one for operation scanning, and one for positioning the patients in the machine room. The two technicians are all protected at level 2 protective standards or above. (2) The patients are asked to wear mask, and the supine position is adopted generally, carry out breathing training for the patients before scanning, and ask them to cooperate with the breathing instructions during the 
examination. Generally, patients should hold the breath at the end of deep inspiration. For severe and critical patients, breath holding is preferred instead of inspiratory requirement. (3) Conventional scanning is performed from the apex of the lung to the costophrenic angle. The scanning direction from costophrenic angle to apex of lung can be adopted in severe patients (those with breath holding difficulties), so as to reduce the respiratory motion artifacts caused by breath holding difficulties in the lower lung field and to ensure the imaging quality. (4) When scanning, use spiral scanning, turn on automatic tube voltage or tube voltage to select 100$120 \mathrm{kv}$, use intelligent milliampere second (50-350 mas), width of collimator at $0.5-1.5 \mathrm{~mm}$, and thickness and spacing of layer at $1-5 \mathrm{~mm}$. Severe and critically ill patients can use large pitch (1.0-1.5 pitch) to reduce scanning time and reduce patient respiratory motion artifacts [10].

\section{Essentials for diagnosis}

Imaging examination was one of the major means for COVID-19 diagnosis, especially the chest CT, which had a high sensitivity for the early diagnosis of COVID-19 [11, 12]. The applications of imaging in COVID-19 included patients screening, comprehensive evaluation, and follow-up.

\section{Imaging features of COVID-19 on chest X-ray}

In the early stage of disease, chest X-ray may be normal. In mild patients with positive reverse-transcription polymerase chain reaction (RT-PCR) results, the main features are unifocal or multifocal patchy opacities in the peripheral and subpleural region of both lungs. With disease progression, there is increasing number of consolidations in both lungs, some of which might coalesce into large consolidation. When the disease continues to develop to a critical stage, it shows diffuse consolidations throughout both lungs, presenting as "white lung" with or without development of pleural effusion [13]. Chest X-ray examination produces a 2-D image of 3-D overlapping structures with limited diagnostic information; therefore, it might not be the best choice for establishing the diagnosis of COVID-19 especially in early stages of the disease.

\section{Imaging features of COVID-19 on chest CT}

Chest CT provides cross-sectional images without overlapping and is often the preferred imaging examination during the outbreak. According to our previous studies, manifestations on CT images are mainly divided into four stages: early stage, progressive stage, severe stage and resorption stage [14-16]. The main imaging features are as follows: (1) early stage: multiple lesions in both lungs are common, while single lesions are rare. Most of the lesions are located in the periphery or subpleural region of the lungs, predominating in the lower lobes. Most of the lesions are irregular and fan-shaped, and they can also be patchy or round-like. Generally, an entire lung segment is not often involved by the lesions. The density of lesions is heterogeneous, usually localized variable sizes patchy ground glass opacities, within which enlarged blood vessels and thick-walled bronchi can be seen to pass through, with or without local interlobular septal thickening. The consolidative area is often small with air bronchogram. (2) Progressive stage: the distribution area of the lesions increases, and the fusion of some lesions expands, which may involve multiple lobes. The density of the lesions increases, and consolidation of different size appear as irregular, wedge-shaped or fan-shaped lesion with unclear boundary, and thickening of the bronchovascular bundle. Multifocal consolidations of both lungs can be seen in the subpleural areas. Lesions progress and change rapidly. The morphology changes greatly on short-term follow-up imaging. Pulmonary tissue necrosis may coalesce to form small cavities. Air bronchogram can be seen. There is usually no pleural effusion. Very few cases are accompanied by enlargement of mediastinal and/or hilar lymph nodes. (3) Severe stage: diffuse lesions can be seen in both lungs. When most of the lung area is involved, it shows "white lung" appearance with elevated diaphragmatic surface. The density of lesions is uneven, and air bronchogram and bronchiectasis are seen within the lesions. The non-solid areas may show patchy ground glass opacities. The interlobar pleura and bilateral pleura may be thickened with a small amount of pleural effusion, presenting as free or loculated effusions. (4) Resorption stage: after isolation and treatment for about one week, most of the patients show improvement, with the extent and number of the lesions decreasing. The density decreases, the consolidation of lung gradually disappears, the ground glass opacities may be completely resorbed, and the exudates may be absorbed. The change of imaging appearance is generally later than the improvement of clinical symptoms. Representative cases of typical COVID-19 appearance on CT are shown in Fig. 4.

\section{Conclusion}

Screening and diagnosis of COVID-19 patients require imaging support. Radiology department staff, especially the radiology technicians, are at high risk of being exposed to COVID-19 patients. Through strict training, assessment and supervision, all medical staff can master the skills of nosocomial infection prevention, and do their own prevention. Insist on wearing masks, strict hand hygiene, and strict implementation of the standard use of protective products. Examine febrile patients with a special machine, 


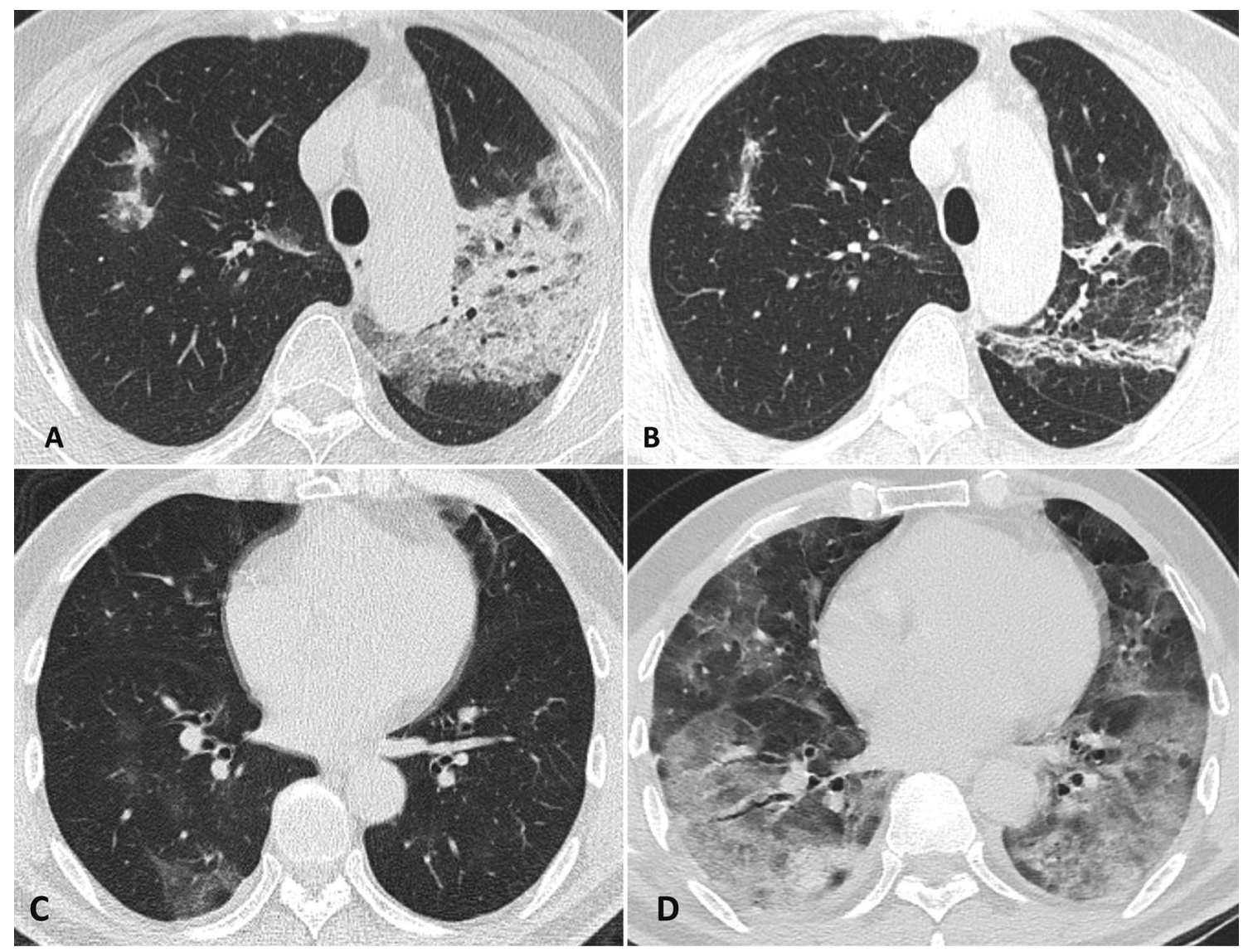

Fig. 4 Two cases of COVID-19 showing the typical CT imaging findings. a, b 70 years old female with bilateral mixed consolidations and ground glass opacities seen on the initial scan (a) that have shown relative decrease in extent and density on the subsequent scan (b). The patient was eventually discharged. c, d 56 years old male with few

and improve the level of protection. Under the premise of ensuring the safety of the patient's examination, keep $1 \mathrm{~m}$ distance from the patient or remotely control to reduce the risk of infection $[6,7]$.

Supplementary Information The online version contains supplementary material available at https://doi.org/10.1007/s42058-021-00055-5.

Acknowledgements The authors would like to express the appreciation for the medical staffs for their efforts to fight against the outbreak.

Author contributions XL and LZ contributed to conception, design, data acquisition, analysis, interpretation and draft of the manuscript; $\mathrm{OA}, \mathrm{ZL}, \mathrm{BL}$ and $\mathrm{HS}$ contributed to data acquisition, analysis and interpretation; $\mathrm{CZ}$ and FY contributed to technical support and design of the research.

\section{Funding None.}

\section{Availability of data and materials Not applicable.}

Code availability Not applicable. patches of ground glass opacities on the initial scan (c). On the subsequent scan acquired in the second week there was a very extensive appearance of ground glass opacities and consolidations throughout both lungs (d) with minimal pleural effusion. The patient situation has worsened and the patient eventually died

\section{Compliance with ethical standards}

Conflict of interest The authors declare no competing interests.

\section{Reference}

1. Zhu N, Zhang D, Wang W, et al. A novel coronavirus from patients with pneumonia in China, 2019. N Engl J Med. 2020;382(8):72733. https://doi.org/10.1056/NEJMoa2001017.

2. Alexander EG, Susan CB, Ralph SB, et al. Severe acute respiratory syndrome-related coronavirus - the species and its viruses, a statement of the Coronavirus Study Group. BioRxiv. https://doi. org/10.1101/2020.02.07.937862. Published online 11 Feb 2020.

3. Zhou P, Yang X, Wang X, et al. A pneumonia outbreak associated with a new coronavirus of probable bat origin. Nature. 2020;579(7798):270-3. https://doi.org/10.1038/s4158 6-020-2012-7.

4. The health industry standard of the People's Republic of China. Technical specification for isolation in hospitals. Published 2019. https://www.whcdc.org/index.php/view/10303.html. Accessed 15 Apr 2020. 
5. National Health Commission of the People's Republic of China. Technical guidelines for the prevention and control of novel coronavirus infection in medical institutions (first edition). Published 2020. http://www.gov.cn/zhengce/zhengceku/2020-01/23/conte nt_5471857.html. 15 Accessed Apr 2020.

6. The health industry standard of the People's Republic of China. Hand hygiene standards for medical staff. Published 2019. http:// www.nhc.gov.cn/wjw/s9496/202002/dbd143c44abd4de8b59a 235feef7d75e/files/6a3e2bf3d82b4ee8a718dbfc3cde8338.pdf. Accessed 15 Apr 2020.

7. The health industry standard of the People's Republic of China. Standard management of environmental surface cleaning and disinfection in medical institutions. Published 2016. http://www.nhc. gov.cn/ewebeditor/uploadfile/2017/01/20170105092341798.pdf. Accessed 15 Apr 2020.

8. National standards of the People's Republic of China. Standard of hospital disinfection. Published 2012. http://www.nhc.gov. cn/ewebeditor/uploadfile/2014/10/20141029163321351.pdf. Accessed 15 Apr 2020.

9. The health industry standard of the People's Republic of China. Technical specifications for disinfection of medical institutions. Published 2012. http://www.nhc.gov.cn/fzs/s7852d/201204/2a75e 255894a4b28827bb996def3cf02.shtml. Accessed 15 Apr 2020.

10. Lei Z, Shi H, Liang B, et al. Imaging examination of pneumonia infected by novel coronavirus (2019-nCoV) and work plan for prevention and control of the epidemic. J Clin Radiol. 2020;39(1):126. https://doi.org/10.13437/j.cnki.jcr.20200206.001.

11. Ai T, Yang Z, Hou H, et al. Correlation of chest CT and RTPCR testing for coronavirus disease 2019 (COVID-19) in China: a report of 1014 cases. Radiology. 2020;296(2):E32-40. https:// doi.org/10.1148/radiol.2020200642.

12. Fan L, Li D, Xue H, et al. Progress and prospect on imaging diagnosis of COVID-19. Chin J Acad Radiol. 2020;18:1-10. https:// doi.org/10.1007/s42058-020-00031-5.

13. Shi H, Han X, Fan Y, et al. Clinical and imaging features of pneumonia infected by novel coronavirus (2019-nCoV). J Clin Radiol. 2020;39(01):8-11. https://doi.org/10.13437/j.cnki.jcr.20200 206.002.

14. Shi H, Han X, Jiang N, et al. Radiological findings from 81 patients with COVID-19 pneumonia in Wuhan, China: a descriptive study. Lancet Infect Dis. 2020;20(4):425-34. https://doi. org/10.1016/s1473-3099(20)30086-4.

15. Pan F, Ye T, Sun P, et al. Time course of lung changes on chest CT during recovery from 2019 novel coronavirus (COVID-19) pneumonia. Radiology. 2020;295(3):715-21. https://doi.org/10.1148/ radiol.2020200370.

16. Jiang N, Zheng C, Fan Y, et al. Subclinical CT imaging features and short-term evolution of COVID-19. Chin J Radiol. 2020;54(04):305-9. https://doi.org/10.3760/cma.j.cn11214920200205-00089.

Publisher's Note Springer Nature remains neutral with regard to jurisdictional claims in published maps and institutional affiliations. 